Journal of Pediatric Gastroenterology \& Nutrition: Publish Ahead Print

DOI: 10.1097/MPG.0000000000002877

\title{
Open Primary Button vs. Laparoscopic Percutaneous Endoscopic Gastrostomy: Results from a Case-Control Study
}

Joseph R Davidson MRCS ${ }^{1,2}$, Do Rae Lee ${ }^{3}$, Dhivya Suresh ${ }^{3}$, Hannah WrightRN(Child) ${ }^{1}$, Manasvi Upadhyaya FRCS ${ }^{1,3}$, Iain E Yardley FRCS ${ }^{1,3}$

1. Department of Paediatric Surgery, Evelina London Children's Hospital, United Kingdom

2. Stem Cells and Regenerative Medicine Section, GOS-UCL Institute of Child Health, London, United Kingdom

3. King's College London School of Medicine, United Kingdom

\section{Correspondence:}

Mr Iain Yardley, Head of Service, Paediatric Surgery Department

Evelina London Children's Hospital, London, United Kingdom, SE1 7EH

Iain.yardley@gstt.nhs.uk

Funding Declaration:JD carried out this work while part-funded as an NIHR Academic Clinical Fellow at the UCL-GOS Institute of Child Health. All research at UCL Great Ormond Street Institute of Child Health is made possible by the NIHR Great Ormond Street Hospital Biomedical Research Centre. The views expressed are those of the author(s) and not necessarily those of the NHS, the NIHR or the Department of Health.

Conflicts of Interest: No author has any conflict of interest to disclose 


\section{Abstract \\ Objectives}

Open primary balloon gastrostomy (PBG) presents an potentialalternative to percutaneous endoscopic gastrostomy (PEG) in children as it obviates the need for change under general anaesthetic (GA), however the complication profile of PBGcompared to PEG is not well defined. Previous series comparing the two have been hampered by the two groups not being equivalent. Our paediatric surgical centre has offeredPBG as an alternative PEG since 2014. We used a matched case-control study to compare outcomes forPBG and PEG.

\section{Methods}

Patients undergoing PBG were used as "cases"and matched 1:3 by age and diagnosis to patients undergoing PEG, demographics and clinical data as "controls". Primary outcome was rate of complications classified according to Clavien-Dindo(I-V). Secondary outcomes included time to feed and length of stay. Non-parametric, categorical analyses and multivariate logistic regression were performed. Data here presented as median [IQR].

\section{Results}

We included 140 patients (35 PBG:105 PEG). The two groups were comparable for gender, weight at surgery and follow-up duration. Median operative time was longer for PBG (43min [IQR 36.5-61.5] vs. 27.5min [18.25-47.75], $\mathrm{p}<0.001)$. Multivariate analysis demonstrated a statistically significant, higher incidence of symptomatic granulation tissue was more common in PBG $(10(29 \%)$ vs $6(6 \%), p=0.0008)$, this remained significant on multivariate analysis (OR 7.56[2.33-23.5], $\mathrm{p}=0.001$ ), nil other complication remained significant nor was overall complication rate statistically different.

\section{Conclusions}

PBG and PEG have similar overall complication rates, however PBG appears to have a higher incidence of granulation tissue. This observation must be weighed against the need for further GA which is not insignificant in medically complex children. 


\section{What is known?}

- Gastrostomy insertion is a common procedure with high rate (up to 45\%) of complications.

- Open Primary Balloon Gastrostomy insertion offers the potential to limit treatment to a single general anaesthetic episode.

- Previous articles comparing complications between insertion techniques are limited by significant differences between patient groups.

\section{What is new?}

- Percutaneous Endoscopic Gastrostomy and Open Primary Balloon Gastrostomy both have an acceptable safety profile (overall complication rate of $33 \%$ )

- Open Primary Balloon Gastrostomy has a significantlyhigher(up toseven-fold increase) rate of symptomatic granulation tissue formation compared to Percutaneous Endoscopic Gastrostomy

- Serious complications may be more common in Open Primary Balloon Gastrostomy(9\% in our series, although not statistically significant)

An infographic is available for this article at: http://links.lww.com/MPG/B902 


\section{Introduction}

Gastrostomy insertion is a commonsurgical procedure,carried out in children across the world. Historically, laparotomy and insertion of a tube using a Stamm technique (or similar) has been performed. More recently, less invasive methods using endoscopy (chiefly percutaneous endoscopic gastrostomy (PEG) utilising a flanged tube), have come to the fore, with the PEG initially described by fellow paediatric surgeons Gauderer, Ponsky and Izant in $1980^{(1)}$. Latterly, fluoroscopy has been employed, opening the field to the domain of interventional radiology (radiologically inserted gastrostomy - RIG).The safety and efficacy of the PEG technique is now well established and addition of laparoscopic to endoscopic visualisation seems to further improve the results ${ }^{(2)}$. However, many children undergoing PEG and RIG procedures will undergo a further procedure under general anaesthetic (GA) to retrieve the flange whenthe tube is first changed. Commonly gastrostomy tubes will be changed in time to a balloon device which will not require further GAs to change. Balloon gastrostomiesmay also be inserted primarily which obviates the need for subsequent anaesthetic, however it requires a larger opening to be made in the stomach wall, subsequently closed around the tube. This differs to the pull-technique used in PEG where the gastric opening matches the size of the tube. This is done with a 'push' technique; either via a mini-laparotomy, laparoscopically where the stomach is grasped and brought to the abdominal wallfor the device to be inserted ${ }^{(3-6)}$, or by an endoscopic approach using devices such as the Cope needle ${ }^{(7,8)}$, or T-fastener ${ }^{(9)}$. These techniques are not necessarily equivalent, as the extent of fixation of the stomach to the abdominal wall may vary, which in turn may affect the risk of complication i.e. displacement of the tube.

Complications from gastrostomy insertion are relatively common, with rates reported as high as $45 \%$ for minor complications and $5 \%$ for major complications ${ }^{(10,11)}$. Granulation tissue, bleeding and infection represent the majority of minor complications. Major complications (those necessitating operative intervention) include tube dislodgement (which can lead to peritonitis) and buried bumper syndrome - which occurs significantly more often with the solid flange of PEG tubes ${ }^{(12)}$. Leakage around the gastrostomy tube is common, but generally inconsequential; however there are instances where severe leakage may lead to marked skin excoriation, necessitating jejunal feeding or potentially a period of parenteral nutrition. 
Gastrostomy may be required in children in whom oral feeding is dangerous (e.g. aspiration risk due to neuromuscular disorder or airway malformation), insufficient (e.g. metabolic disorder or feed aversion) or even impossible (e.g. oesophageal atresia). Clearly therefore the cohort of children undergoing gastrostomy insertion are diverse in terms of their underlying conditions. This diversity in patient groups has meant that studies into the most suitable technique for gastrostomy insertion have been limited, and Singh and colleagues have addressed this with the only randomised trial in children to date compared PEG with RIG at a single tertiary centre $^{(13)}$.The largest paediatric retrospective series from Miyata and colleagues have demonstrated similar complication rates between PEG and PBG insertion ${ }^{(14)}$ but, in common with other comparative reports,studied unmatched patient groups.

Our tertiary centre routinely offersboth PEG andopen primary balloon gastrostomy. This study aimed to compare and contrast the outcomes of the two approachesusing a matched case-control study methodology to generate comparable patient groups.

\section{Methods}

\section{$\underline{\text { Study Design }}$}

This study was performed as an audit of clinical practice and institutional ethical approval was given (Local Study ID 10019). The study was conducted in a tertiary paediatric surgical centre and patients younger than 18 years of age were included. All open primary balloon gastrostomy (PBG) inserted from the introduction of the procedure to the time of the study (April 2015 November 2019) were included. Patients receiving PBG,did so according to surgeon and family preference. These patients were considered "cases" and were matched 1:3 by age and primary diagnosis with PEG insertions from the same department over the same time period ("controls"). Primary diagnosis was determined by the specialist team referring for the gastrostomy: Neurodisability - patients had uncoordinated swallow due to an underlying neurological condition and needed tube-assisted feeding. Metabolic - children needed gastric access for overnight feeding or rescue medication. Anatomical risk - referred due to aspiration risk in a child with anatomical airway anomaly (e.g. laryngeal cleft or pharyngeal malformation). Behavioural - referred due to feeding issues secondary to behavioural issues e.g. severe autism. 
Controls were selected to match diagnostic category and age and were assessed for similarity of body weight and BMI.We excluded any PBG procedures performed for patients with long-gap oesophageal atresia as no matched controls were available. Selection of controls was blind to their outcomes and was performed by researchers (DRL and DS) not involved in the clinical care of patients. Primary outcome was incidence of major complications ( $\geq$ Grade III, defined according to Clavien-Dindo classification ${ }^{(15)}$ ), secondary outcomes were operative time, time to full feeds, incidence of minor complications (Grade I+II), and need for further planned procedures (i.e. tube change).

\section{Operative Technique}

PEG is performed by all surgeonsat our institution with additional laparoscopic visualisation via a $5 \mathrm{~mm}$ camera inserted at the umbilicus. Peritoneal insufflation is reduced and the laparoscopic light switched off to allow visualisation of the puncture of the stomach by the percutaneous needle. A 9Fr or $15 \mathrm{Fr}$ flanged tube (Freka ${ }^{\circledR}$ PEG Gastric Set EnFIT) is then inserted by the standard "pull technique" as has been welldescribed in both children and adults ${ }^{(16)}$. Feeding is commenced at the surgeon's discretion.

In our centre a single surgeon (MU) performs PBG via a transverse incision $(2 \mathrm{~cm})$ over the upper left rectus abdominis. On entering the abdomen, the stomach is identified and stay sutures placed in the posterior rectus sheath. An appropriately sized button gastrostomy (Mini Balloon Button, GBUK Enteral Ltd.) is inserted in the centre of a double purse-string of polyglactin (short lived absorbable) sutures of in the anterior aspect of the junction between the gastric antrum and body. The stomach is sutured to the posterior rectus sheath with polydioxanone (long lasting absorbable) sutures and then a layered closured is performed. The device is secured to the skin with prolene (inert, nonabsorbable suture) sutures for one week post-operatively. Feeding is routinely commenced on the first post-operative day.

\section{$\underline{\text { Data Collection }}$}

Data on patient demographics, comorbidities, complications, and further procedureswere collected from the patient clinical record. Surgical time and time under GA was retrieved from our theatre management system. Time to full feeds and length of stay were rounded to the 
nearest whole day. All patients were followed up by a specialist gastrostomy nurse who maintained contact with families and kept a prospective log of all post-operative complications.

Complications were scored according to the validated and widely adopted Clavien-Dindo classification system as follows $^{(15)}$ : I = deviation from post-operative course, II = initiation of pharmacological therapy, III = necessitating invasive intervention, $\mathrm{IV}=$ requiring $\mathrm{ICU}$ admission, $\mathrm{V}=$ Death) by two independent experts, and then re-reviewed following identification of discrepant cases. Specific incidence of certain complications were noted. Infection was defined as a case where prescription antibiotics (including topical antibiotics) were administered, formation of granulation tissue was defined as tissue requiring intervention(either topical treatment such as silver nitrate or steroids or surgical excision), leak was defined asa leakage of feed at the site of the gastrostomy, a tube displacement was defined as a tube which had completely fallen out.

Acknowledging that many children with PEG would need a second procedure under GA in order to change / remove the device, we noted complications at the initial procedure and cumulative incidence with subsequent procedures. We excluded insertion of gastrojejunal tubes from 'further procedures under GA' as this was regarded a patient-specific requirement rather than a device specific one and would typically require a GA at our institution.

\section{$\underline{\text { Statistical Analysis }}$}

Statistical analyses were performed using Prism 8 for MacOS (v8.3.0, October 2019, GraphPad Software LLC) and SPSS 26 (v26.0.0.0, 2019, IBM SPSS Statistics.) Continuous variables are described with median[IQR] and compared using Mann-Whitney U test. Categorical data are compared with Fisher's Exact or Chi-square test. Multivariate analysis was performed to control for age, weight and diagnosis when exploring the association of outcome measures with gastrostomy device. Exact p-values for Fisher's Exact are reported, and to 3 significant figures down to $<0.001$ for Mann-Whitney; statistical significance was accepted at $\mathrm{p}<0.05$. 


\section{Results}

$\underline{\text { Patient cohort, operative and admission details }}$

We had complete data on all 35 PBGperformed at our institution over the past 3 years. These were successfully matched 1:3 with PEG controls by age and diagnosis, with comparable weight at surgery and duration of follow-up noted (Table 1.) PBG had a longer surgical time (43min [36.5-61.5] vs. 27.5min [18.25-47.75], $\mathrm{p}<0.001)$, a longer time under GA (150min [116.75185.75 ] vs. $123.5 \mathrm{~min}$ [92.25-154], $\mathrm{p}=0.02$ )and a longer time to reaching full feeds(1day [0-1] vs. 0 days $[0-1], \mathrm{p}=0.004)$; however there was no statistically significant difference in length of stay (2 days [1-4] vs. 1 day [1-3], $\mathrm{p}=0.079$ ).

\section{$\underline{\text { Post-operative Complications }}$}

Inter-observer concordance for grading of complications was $88 \%$, with perfect consensus on a second review of cases.Overall complication rate was 33\%(Table 2), with complications reported in 16 PBG (46\%) and 30 PEG insertions (29\%), which increased to 34(32\%) when tube changes were included. Chi-square test for trend between the two techniques showed no statistical difference $\left(c^{2}=5.4, p=0.23\right)$. Specific complications of infection, formation of granulation tissue, leak and tube dislodgement are noted in Table 3. Major surgical complications were noted in 5 patients. 2/35 (6\%)PBG patients needed to return to theatre, at 2 and 14 days respectively, for dislodged devices on the ward. Both these patients developed peritonitis and required a laparotomy and reinsertion of the balloon gastrostomy, one of them was admitted to intensive care following the emergency surgery. 1 patient with PEG had to return to theatre from recovery for port site bleeding, a further patient with PEG required a mini-laparotomy for buried bumper 56 months after the initial insertion having been lost to follow up. The remaining Grade 3 complication was a PBG patient experiencing severe wound breakdown and requiring GA to obtainintravenous access.

Perceiving the complication rate of PBG to be higher than expected, we performed post-hoc analysis to examine the effect of a learning curve effect on complications. We compared the initial 15 cases to the subsequent 20 cases. The overall complication rate was significantly lower in the later group ( $66 \%$ vs $25 \%, \mathrm{p}<0.05$ ) as was the formation of granulation tissue ( $47 \%$ vs. $15 \%, \mathrm{p}<0.05)$. Leakage ( $20 \%$ vs $5 \%, \mathrm{p}=\mathrm{NS})$ and site infection ( $33 \%$ vs $10 \%, \mathrm{p}=\mathrm{NS})$ were also 
both less frequent but not statistically significantly so. Major complications were reported in both groups (7\% vs 10\%) and both dislodged tubes occurred in the most recent 10 cases.

\section{$\underline{\text { Further procedures }}$}

7 patients with PEG and 2 with PBG had conversion of their gastrostomy to a gastrojejunal feeding tube. Of the remaining 98 PEG patients, 44(45\%) had a change of gastrostomy tube (PEG-PEG or PEG-BG) under GA at a median 12 months [7-23] post-primary procedure. Median operative time for these procedures was 13 minutes [7.5-20.5], GA time was 88 minutes [70-117.5]. There were two grade I and four grade II complications from changes of device (2 infection, 4 granuloma). Two patients' PEG tubes were changed to gastrostomy buttons using an awake "cut-and-push" technique ${ }^{(17)}$. There were no anaesthetic complications in any patient.

\section{Multivariate analysis}

Multivariate binary logistic regression were performed to assess the independent influence of gastrostomy type on complications (overall and $\geq$ Grade III) as well as specific incidence of granulation tissue, site infection and leakage from the gastrostomy.Other variables in the model were age, gender, weight, diagnosisand duration of follow-up. There were no significant effect of any of these variables on multivariate analysis for complications overall nor specific incidence of granulation, infection, leak or Grade III-V complication. Effect direction and associated confidence intervalsare demonstrated in Figure 1. Symptomatic granulation tissue requiring treatment was more common in patients with PBG (OR 7.56[2.33-23.5], p=0.001). Any complication (OR 2.17[0.97-4.83], p=0.059) and Grade III-V complication (OR 5.02[0.73-34.4], $\mathrm{p}=0.10$ ) were not statistically different between gastrostomy types.

\section{Discussion}

This case control study explores the surgical outcomes from primary balloon gastrostomy and PEG, controlling for the differences in patient demographics commonly observed in retrospective cohort studies. The complication rate in the cohort was 33\%(46/140)for minor and

$4 \%(5 / 140)$ for major complications, which aligns with published literature ${ }^{(2,11)}$.Within our cohort the complication rate was higher for primary PBG compared to PEG and although some results 
did not attain statistical significance due to wide confidence intervals, these may yet be clinically significant and important to consider. The development of symptomatic granulation tissue at the gastrostomy site was significantly higher in PBG, perhaps due to the need for a formal gastrotomy or increased movement of the gastrostomy device post-operatively.We report two cases with dislodged balloon devices, both were related to balloon deflation and subsequent tube dislodgement; this may not be a surgical complication but should be acknowledged as an inherent risk of the insertion of a balloon device into a newly formed gastrostomy and has been described as a risk unique to $\mathrm{PBG}^{(18)}$, although the rate has been reported considerably higher in other series where an endoscopic approach is used for insertion ${ }^{(19)}$. We hypothesise that the open approach may result in a more secure fixation of the device by anchoring the stomach to the abdominal wall. There were no cases of gastrocolic fistula, which can be attributed to direct visualisation of the puncture site of the stomach - either by laparoscopy in the PEG group or through the open technique used for balloon device insertion - this is not an insignificant result since the incidence of gastrocolic fistula in paediatric PEG insertion can be as high as $4 \%{ }^{(18)}$.

There are clearly risks associated with both surgical approaches described here, however the risk of general anaesthetic is not something which has been readily acknowledged in many surgical studies. Furthermore, there is ongoing uncertainty of the long-term effects of general anaesthetic agents in young children on neurodevelopmental outcome ${ }^{(20)}$, this continues to merit consideration as studies such as the GAS Randomised Control Trial had stringent inclusion criteria that do not reflect the patient population in question here ${ }^{(21)}$. A single GA episode may be attractive in children with metabolic disorders in whom supervised fasting isrequired (such as $9 \%$ of our patient cohort), in patients for whom GA is hazardous, for example those with complex congenital cardiac disease, or in the considerable number of children who may have challenges coping with hospital admissions due to anxiety ${ }^{(22)}$. Therefore all of these patient groups could be considered for primary balloon device insertion and are offered PBG at our centre.

Many previous studies, including those referenced here, have been unable to control for patient variables when assessing complication rates in retrospective studies of different gastrostomy devices and tube insertion techniques ${ }^{(14,19,23-27)}$. By using a matched case-control study methodology we have been able to control for some of this variability and create comparable 
patient groups.However, our study was limited by a lack of randomisation and the possibility of inherent differences between the two groups that are unmeasured. A single surgeon carried out all PBG procedures creates the possibility of surgical technique or aptitude affecting the results. It is unclear if the primary diagnosis leading to gastrostomy insertion affects the complications rate, it is possible that either PEG orPBG may be preferred in certain conditions. Finally, our length of follow up is finite and so we can only report early and medium-term complications; while the majority of complications are self-evident within the time period studied, it may be that there are significant differences in rates of complications emerging later. Further larger, longer and preferably multicentre, studies are required to explore these issues to better define the utility of the two conceptsand also to investigate the optimum method of insertion.

Our studyconfirms that complication rates of PBG are acceptable when compared to PEG and the avoidance of further GAmeans this continues to be the insertion technique of choice for those patients at particularly high risk when undergoing further procedures under GA. As such we continue to consider both procedures for patients needing gastrostomy at our centre.

\section{References}

1. Gauderer MWL, Ponsky JL, Izant RJ. Gastrostomy without laparotomy: A percutaneous endoscopic technique. J Pediatr Surg 1980; 15:872-875.

2. Baker L, Beres AL, Baird R. A systematic review and meta-analysis of gastrostomy insertion techniques in children. J Pediatr Surg 2015; 50:718-25.

3. Georgeson KE. Laparoscopic fundoplication and gastrostomy. Semin Laparosc Surg $1998 ; 5: 25-30$.

4. Vasseur Maurer S, Reinberg O. Laparoscopic technique to perform a true Stamm gastrostomy in children. J Pediatr Surg 2015; 50:1797-1800.

5. McCagg J, Markham S, Idowu O, et al. Modification of U-Stitch Laparoscopic Gastrostomy Technique to Minimize Suture Knot Abscess Formation. Eur J Pediatr Surg $2015 ; 26: 252-254$. 
6. Villalona GA, Mckee MA, Diefenbach KA. Modified Laparoscopic Gastrostomy Technique Reduces Gastrostomy Tract Dehiscence. J Laparoendosc Adv Surg Tech 2011; 21:355-359.

7. Griffiths M. Single-Stage Percutaneous Gastrostomy Button Insertion: A Leap Forward. $J$ Parenter Enter Nutr 1996; 20:237-239.

8. Darwish AA, Corbett P, Louson A, et al. Single stage Percutaneous Rapid INsertion of a Gastrostomy button (SPRING) technique: A retrospective study in children. J Pediatr Endosc Surg 2020.

9. Kvello M, Knatten C, Perminow G, et al. Initial experience with percutaneous endoscopic gastrostomy with T-fastener fixation in pediatric patients. Endosc Int Open 2018; 06:E179-E185.

10. Sealock RJ, Munot K. Common Gastrostomy Feeding Tube Complications and Troubleshooting. Clin Gastroenterol Hepatol 2018; 16:1864-1869.

11. Balogh B, Kovács T, Saxena AK. Complications in children with percutaneous endoscopic gastrostomy (PEG) placement. World J Pediatr 2019; 15:12-16.

12. Cyrany J, Rejchrt S, Kopacova M, et al. Buried bumper syndrome: A complication of percutaneous endoscopic gastrostomy. World J Gastroenterol 2016; 22:618-27.

13. Singh RR, Nah SA, Roebuck DJ, et al. Double-blind randomized clinical trial of percutaneous endoscopic gastrostomy versus radiologically inserted gastrostomy in children. Br J Surg 2017; 104:1620-1627.

14. Miyata S, Dong F, Lebedevskiy O, et al. Comparison of operative outcomes between surgical gastrostomy and percutaneous endoscopic gastrostomy in infants. J Pediatr Surg 2017; 52:1416-1420.

15. Dindo D, Demartines N, Clavien P-A. Classification of surgical complications: a new proposal with evaluation in a cohort of 6336 patients and results of a survey. Ann Surg 2004; 240:205-13. 
16. Abbassi Z, Naiken SP, Buchs NC, et al. Laparoscopic-assisted percutaneous endoscopic gastrostomy in two patients who failed percutaneous endoscopic gastrostomy. Int J Surg Case Rep 2015; 13:40-2.

17. Thomas H, Yole J, Livingston MH, et al. Replacing gastrostomy tubes with collapsible bumpers in pediatric patients: Is it safe to "cut" the tube and allow the bumper to pass enterally? J Pediatr Surg 2018; 53:942-945.

18. Landisch RM, Colwell RC, Densmore JC. Infant gastrostomy outcomes: The cost of complications. J Pediatr Surg 2016; 51:1976-1982.

19. Baker L, Emil S, Baird R. A comparison of techniques for laparoscopic gastrostomy placement in children. J Surg Res 2013; 184:392-396.

20. Walkden GJ, Pickering AE, Gill H. Assessing Long-term Neurodevelopmental Outcome Following General Anesthesia in Early Childhood: Challenges and Opportunities. Anesth Analg 2019; 128:681-694.

21. Davidson AJ, Disma N, de Graaff JC, et al. Neurodevelopmental outcome at 2 years of age after general anaesthesia and awake-regional anaesthesia in infancy (GAS): an international multicentre, randomised controlled trial. Lancet (London, England) 2016; 387:239-50.

22. Eijlers R, Utens EMWJ, Staals LM, et al. Systematic Review and Meta-analysis of Virtual Reality in Pediatrics: Effects on Pain and Anxiety. Anesth Analg 2019; 129:1344-1353.

23. Mason CA, Skarda DE, Bucher BT. Outcomes after laparoscopic gastrostomy suture techniques in children. J Surg Res 2018; 232:26-32.

24. Aprahamian CJ, Morgan TL, Harmon CM, et al. U-stitch laparoscopic gastrostomy technique has a low rate of complications and allows primary button placement: Experience with 461 pediatric procedures. J Laparoendosc Adv Surg Tech 2006; 16:643649.

25. Merli L, De Marco EA, Fedele C, et al. Gastrostomy Placement in Children. Surg Laparosc Endosc Percutan Tech 2016; 26:381-384. 
26. Bentley VL, Seemann NM, Blackmore C. A comparison of balloon and nonballoon gastrostomy tubes in children. J Pediatr Surg 2020; 55:852-854.

27. Göthberg G, Björnsson S. One-Step Insertion of Low-Profile Gastrostomy in Pediatric Patients vs Pull Percutaneous Endoscopic Gastrostomy. J Parenter Enter Nutr 2016; 40:423-430.

Figure Legends

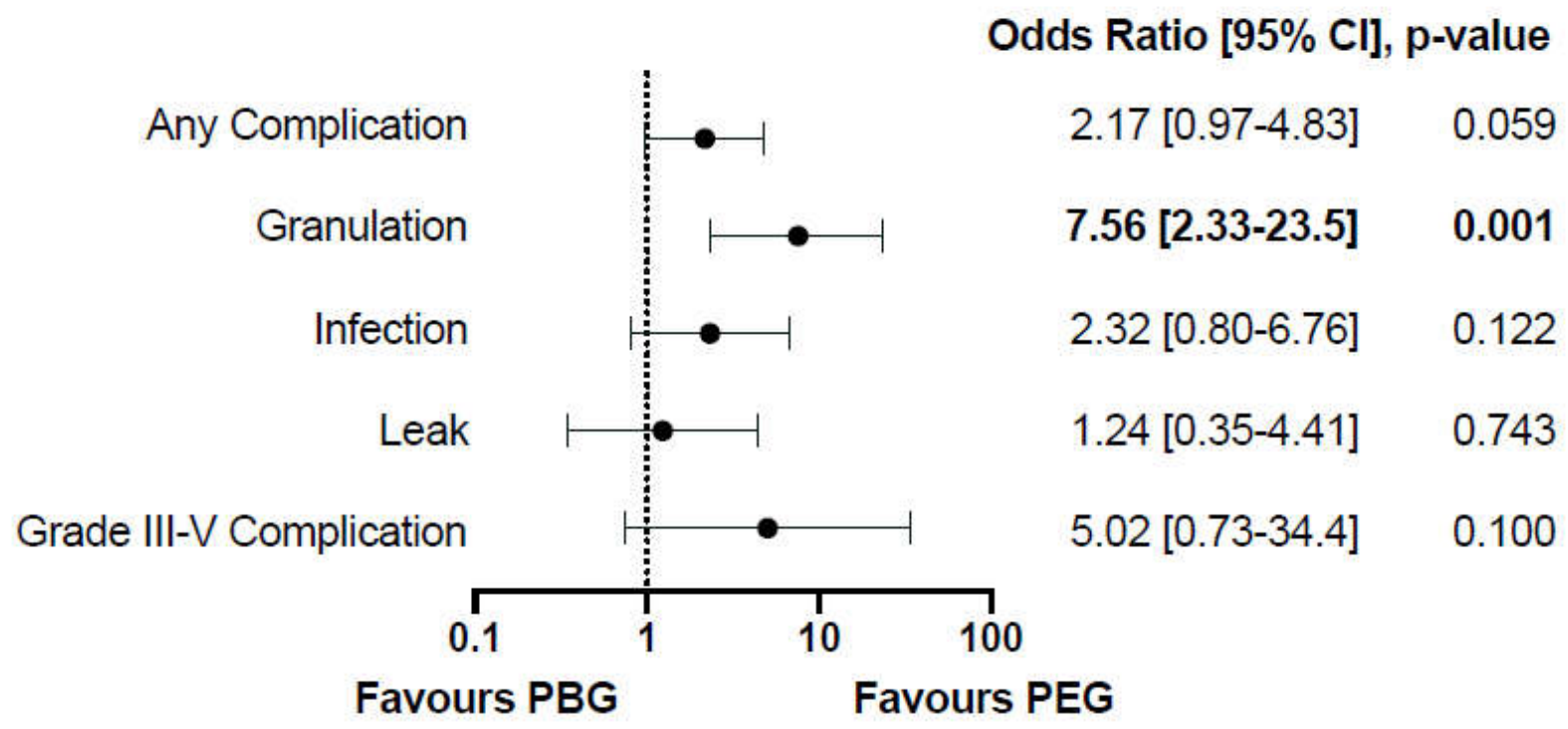

Figure 1Multivariateanalysis comparing the effects of PBG/PEG on different outcomes (model controlled for age, weight, gender, diagnosis and duration of follow-up) 
Table 1 Study Group Demographics, Admission and Operative Details (median [IQR]), MannWhitney U Test performed.

\begin{tabular}{|c|c|c|c|}
\hline & $\begin{array}{l}\text { Primary Button } \\
(\mathrm{n}=35)\end{array}$ & $\begin{array}{l}\text { Laparoscopic PEG } \\
(\mathrm{n}=105)\end{array}$ & $\mathrm{p}, \mathrm{M}-\mathrm{WU}$ \\
\hline Age (months) & $17[10-36]$ & $19[12-31.5]$ & - \\
\hline Sex (Female/Male) & $13 / 22$ & $50 / 55$ & 0.33 \\
\hline Weight (kg) & $9.12[7.7-13.9]$ & $10.14[7.29-13.75]$ & 0.998 \\
\hline \multicolumn{4}{|l|}{ Diagnosis } \\
\hline Neurodisability & $13(37 \%)$ & $39(37 \%)$ & \\
\hline Metabolic & $3(9 \%)$ & $9(9 \%)$ & - \\
\hline Anatomical risk & $15(43 \%)$ & $45(43 \%)$ & - \\
\hline Behavioural & $4(11 \%)$ & $12(11 \%)$ & - \\
\hline \multicolumn{4}{|l|}{ Operative + Admission } \\
\hline \multicolumn{4}{|l|}{ Details } \\
\hline Surgical Time (min) & $43[36.5-61.5]$ & $27.5[18.5-47.75]$ & $<0.001$ \\
\hline Time under GA (min) & 150 & $123.5[92.25-154]$ & 0.02 \\
\hline Time to full feeds (days) & $1[0-2]$ & $0[0-1]$ & 0.004 \\
\hline Length of Stay (days) & $2[1-4]$ & $1[1-3]$ & 0.079 \\
\hline Time of follow up (months) & $16[7-20]$ & $11.5(6-27)$ & 0.73 \\
\hline
\end{tabular}


Table 2. Surgical Complications by Grade - each patient represented once, by highest grade of complication. (Clavien-Dindo: I = deviation from post-operative course, II = initiation of pharmacological therapy, III = necessitating invasive intervention, IV = requiring ICU admission, $\mathrm{V}=$ Death), Fisher's Exact performed, NS = non-significant. Chi-square test for trend between PBG and PEG

\begin{tabular}{|llllll|}
\hline & Primary & \multicolumn{2}{l}{ Laparoscopic PEG $(\mathrm{n}=105)$} & Overall & Chi- \\
& Button & Insertion & All & $(\mathrm{n}=140)$ & square for \\
& $(\mathrm{n}=35)$ & & Procedures & & trend \\
\hline No Complication & $19(54 \%)$ & $75(71 \%)$ & $71(68 \%)$ & $90(64 \%)$ & \\
C-D I & $5(14 \%)$ & $11(10 \%)$ & $11(10 \%)$ & $16(11 \%)$ & \\
C-D II & $9(26 \%)$ & $18(17 \%)$ & $22(21 \%)$ & $31(22 \%)$ & $\mathrm{p}=0.23$ \\
C-D III & $2(6 \%)$ & $2(2 \%)$ & $2(2 \%)$ & $2(1 \%)$ & \\
C-D IV & $1(3 \%)$ & - & - & $1(1 \%)$ & \\
C-D V & - & - & - & & \\
\hline
\end{tabular}

Table 3. Rates of site infection, granulation tissue, tube leaking and displacement for primary gastrostomy insertion (possible more than one in same patient). Fisher's Exact performed, NS = non-significant

\begin{tabular}{|c|c|c|c|}
\hline \multicolumn{4}{|l|}{ Specific Complications of Primary Surgery } \\
\hline Primary Button $(n=35)$ & $\begin{array}{l}\text { Laparoscopic } \\
(\mathrm{n}=105)\end{array}$ & PEG & Fisher's Exact, $\mathrm{p}$ \\
\hline Infection $\quad 7(20 \%)$ & $10(10 \%)$ & & 0.130 \\
\hline Granulation tissue $\quad 10(29 \%)$ & $6(6 \%)$ & & 0.0008 \\
\hline $4(11 \%)$ & $10(10 \%)$ & & 1 \\
\hline Dislodged & 0 & & 0.061 \\
\hline
\end{tabular}

